\title{
Một thuật toán tối ưu bám quỹ đạo mục tiêu của bài toán quan sát đa mục tiêu trong trường hợp có mục tiêu bị che khuất
}

\author{
Nguyễn Thị Hằng \\ Trường Đại học Mỏ - Địa chất, Hà Nội \\ E-mail: nguyenthihang@humg.edu.vn \\ Ngày nhận bài: 09/05/2019, ngày sửa chữa: 13/09/2019, ngày duyệt đăng: 13/09/2019 \\ Xem sớm trực tuyến: 13/09/2019, định danh DOI: 10.32913/mic-ict-research-vn.v2019.n1.861 \\ Biên tập lĩnh vực điều phối phản biện và quyết định nhận đăng: TS. Nguyễn Việt Dũng
}

Tóm tắt: Trong thực tế quan sát quỹ đạo đa mục tiêu di động, có lúc hệ thống quan sát không thể nhận biết được mục tiêu, do các mục tiêu chuyển động quá gần nhau trong khi độ phân giải của hệ thống quan sát bị hạn chế, hoặc do một số mục tiêu bị che khuất bởi các mục tiêu khác vì một lý do quan trắc nào đó. Trường hợp này cũng thường xảy ra trong những môi trường có số lượng mục tiêu lớn (dày đặc) và mật độ nhiễu lớn. Các thuật toán bám mục tiêu, bám quỹ đạo hiện hành gặp khó khăn và thường mất bám, mất quỹ đạo bám. Trong bài báo này, chúng tôi trình bày một phương pháp liên kết dữ liệu và thuật toán bám quỹ đạo đệ quy từng bước theo thời gian quan sát với sự sử dụng tối đa dữ liệu lịch sử của quỹ đạo. Thuật toán khắc phục được tình trạng mất bám, mất quỹ đạo bám trong môi trường có mục tiêu bị che khuất. Thuật toán là sự kết hợp tư tưởng của phương pháp liên kết dữ liệu đa giả thiết và lọc Kalman mở rộng. Bài báo cũng chứng minh sự tồn tại của lời giải tối ưu từng bước và đưa ra thuật toán tìm lời giải $\varepsilon$-tối ưu.

Từ khóa: Mục tiêu, quỹ đạo, ảnh, bám muc tiêu, quỹ đạo bám, che khuất, dây chuyền, dây chuyền dũ liệu.

$\begin{aligned} & \text { Title: } \text { An Optimal Algorithm for Multi-Target Tracking with Obscured Targets } \\ & \text { Abstract: } \text { In multiple-target tracking, there are difficult cases that the tracking system cannot detect targets, that is when targets } \\ & \text { move too closely to each other beyond the resolution of the tracking system, or some targets are possibly obscured } \\ & \text { by others. This also happens in environments with a large number of targets. In such cases, state-of-the-art tracking } \\ & \text { algorithms fail to track targets or their orbits. In this paper, we propose a data association tracking method and } \\ & \text { corresponding recursive tracking algorithm taking into account as many past orbit data as possible. This algorithm is } \\ & \text { able to track targets and orbits in cases of obscured targets. This algorithm combines the data association method of } \\ & \text { multiple hypothesis tracking and the extended Kalman filtering. In addition, we also prove the existence of the optimal } \\ & \text { tracking solution at each step and give the algorithms for finding the } \varepsilon \text {-optimal solution. } \\ & \text { Target, orbit, image, target tracking, orbit tracking, obscured, chain, data transmission. }\end{aligned}$

\section{GIỚI THIÊ̂U}

Mô hình quan sát đa mục tiêu di động (MTT: MultipleTarget Tracking) được ứng dụng rộng rãi trong thực tiễn hoạt động xã hội, trong nhiều lĩnh vực cả ở dân sự lẫn trong quân sự. Trong dân sự, các mô hình đã và đang được ứng dụng như: hệ thống điều khiển và giám sát không lưu, hệ thống điều khiển giao thông, hệ thống giám sát đại dương, hệ thống bảo vệ và giám sát người qua lại trong một vùng được bảo vệ. Trong quân sự, các mô hình đã và đang được áp dụng như: hệ thống radar phòng thủ tên lửa đạn đạo, hệ thống phòng không, hệ thống giám sát vùng mục tiêu bảo vệ nào đó, hệ thống giám sát và theo dõi phòng không.
Công cụ vật lý được sử dụng trong các hệ thống quan sát có thể là video, radar, hay cảm biến (sensor) nào đó. Công cụ toán học (phần hồn của hệ thống) được sử dụng cho đến thời điểm hiện tại là các kết quả, các thuật toán nghiên cứu về MTT.

Phương pháp toán học phổ biến để giải bài toán MTT là phương pháp ước lượng tuần tự Bayes (Bayesian Sequential Estimation). Phương pháp này về bản chất là cập nhật một cách đệ quy hàm phân bố hậu nghiệm các trạng thái của mục tiêu. Tất cả các thuật toán xây dựng trên nguyên tắc này cho đến thời điểm hiện tại được công bố đều là các thuật toán không tầm thường vì nó được gắn với các mô hình xác suất rất phức tạp. 
Các thuật toán chính hiện hành bao gồm: thuật toán lân cận gần nhất toàn cục (GNN: Global Nearest Neighbors) [1-3], thuật toán kết hợp dữ liệu xác suất đồng thời (JPDA: Joint Probabilistic Data Association) [4-6], thuật toán kết hợp dữ liệu đa giả thiết (MHT: Multiple Hypothesis Tracking) [7-10], thuật toán kết hợp dữ liệu xác suất đồng thời gần nhất (NNJPDA: Nearest Neighbor Joint Probabilistic Data Association) [11, 12]. Các thuật toán này rất hiệu quả, đã và đang được sử dụng trong thực tế. Ví dụ, hệ thống giám sát điều khiển không lưu (hệ radar ASDE-X) sử dụng thuật toán JPDA, hệ radar mảng pha Cobra Dane nhằm phát hiện và giám sát tầm xa các tên lửa đạn đạo xuyên lục địa sử dụng thuật toán NNJPDA kết hợp với bộ lọc Kalman mở rộng (EKF: Extended Kalman Filter), hệ thống radar trên biển X-band (SBX) của Hải quân Mỹ cũng sử dụng thuật toán NNJPDA kết hợp với bộ lọc EKF, hệ thống radar mảng pha cảnh báo sớm (UEWR: Upgraded Early Warning Radar) nằm trong hệ thống phòng thủ tên lửa quốc gia Mỹ sử dụng thuật toán MHT kết hợp với bộ lọc EKF, hệ thống radar THAAD sử dụng thuật toán JPDA cổ điển kết hợp với bộ lọc EKF, hệ thống video giám sát hoạt động con người trong một vùng bảo vệ (của Đức) dùng thuật toán MHT.

Thuật toán MHT được đề xuất bởi Reid còn thuật toán JPDA được đề xuất bởi Bar-Shalom. Song từ khi được đề xuất cho đến các cài đặt trong ứng dụng hiện nay, đã có nhiều nhà toán học nghiên cứu, bố sung và phát triển so với các đề xuất ban đầu. Động lực của các nghiên cứu bổ sung và phát triển đó là: đặc thù của các đối tượng quan sát, đặc thù của mô hình quan sát và đặc biệt là sự phát triển của các công cụ vật lý - các công cụ "giá mang" và "nền tảng kỹ thuật" của các thuật toán đó.

Tuy nhiên, các thuật toán hiện hành đối với bài toán MHT chưa được giải quyết triệt để một tồn tại mà bài báo này hướng tới để giải quyết, đó là mô hình MTT với hiện tượng mục tiêu bị che khuât. Trong thực tế quan sát quỹ đạo đa mục tiêu di động, có lúc các mục tiêu chuyển động quá gần nhau trong khi độ phân giải của hệ thống quan sát bị hạn chế, hoặc do một lý do quan trắc nào đó mà một số mục tiêu bị che khuất bởi các mục tiêu khác. Các tình huống này làm cho không phát hiện được mục tiêu. Các thuật toán bám mục tiêu, bám quỹ đạo hiện hành gặp khó khăn và thường mất bám, mất quỹ đạo bám. Trường hợp này cũng thường xảy ra trong những môi trường có số lượng mục tiêu lớn (dày đặc) và mật độ nhiễu lớn.

Bài báo này trình bày một số kết quả mới về bài toán MTT trong điều kiện tổng quát, đặc biệt khi hiện tượng mục tiêu bị che khuất có thể xảy ra ${ }^{1}$. Trước hết, chúng tôi

\footnotetext{
${ }^{1}$ Một phần của các kết quả này đã được trình bày tại hội nghị khoa học quốc tế "Vietnam International Applied Mathematics Conference (VIAMC)" vào tháng 12/2017 và tại hội thảo khoa học về "Một số phương pháp thống kê hiện đại và các ứng dụng" vào tháng 07/2019.
}

đưa ra phương pháp liên kết dữ liệu thông qua hệ thống ánh xạ xác định đệ quy từng bước. Hệ thống ánh xạ này không chỉ quan tâm tới bản thân số liệu quan sát mà còn tính đến cả lịch sử quỹ đạo quá khứ có thể có của số liệu đó. Bởi vậy phương pháp liên kết dữ liệu này khắc phục được hiện tượng mục tiêu bị che khuất (nếu xảy ra) và không làm mất mục tiêu, mất quỹ đạo bám, v.v. Tiếp đến, dựa vào ý tưởng và quan điểm của thống kê Bayes, chúng tôi đưa ra khái niệm lời giải tối ưu từng bước theo nghĩa làm cực đại xác suất hậu nghiệm tại mỗi bước, cũng như chứng minh sự tồn tại lời giải tối ưu từng bước đối với phương pháp liên kết dữ liệu đề xuất. Cuối cùng, dựa vào phương pháp dùng lọc Kalman để ước lượng quỹ đạo của mục tiêu trên cơ sở dữ liệu quan sát, chúng tôi đưa ra khái niệm lời giải $\varepsilon$-tối ưu. Bản chất của khái niệm này là, khi dùng dữ liệu quan sát của dây chuyền dữ liệu ảnh, theo phương pháp ước lượng của lọc Kalman để ước lượng quỹ đạo của mục tiêu thì phương sai $P(t \mid t)$ không vượt quá $\varepsilon$ (cho trước tùy ý bé) với mọi $t$ và đối với mọi quỹ đạo của mọi mục tiêu được quan tâm trong bài toán MTT. Với khái niệm đó, chúng tôi đã đưa ra thuật toán xây dựng lời giải $\varepsilon$-tối ưu mà bản chất là xây dựng hệ thống ánh xạ liên kết dữ liệu đã nêu. Trong khuôn khổ giới hạn của bài báo, chúng tôi chỉ trình bày chi tiết các bước của thuật toán và sơ đồ logic cài đặt chi tiết, mà chưa đề cập đến mô phỏng và áp dụng cho một ứng dụng thực tiễn cụ thể.

Cấu trúc tiếp theo của bài báo như sau. Mục II trình bày mô hình toán học của bài toán cùng các khái niệm và kết quả bổ trợ ban đầu. Mục III là về khái niệm lời giải tối ưu từng bước và sự tồn tại lời giải tối ưu từng bước. Mục IV xây dựng thuật toán đệ quy để tìm lời giải $\varepsilon$-tối ưu. Mục $\mathrm{V}$ và VI là phần thảo luận và kết luận.

\section{BÀI TOÁN QUAN SÁT ĐA MỤC TIÊU}

Giả sử ta cần quan tâm đến một số đối tượng (hay còn gọi là mục tiêu) di động nào đó trong một miền không gian và trong một khoảng thời gian nào đó. Ký hiệu $\mathcal{R}$ là miền không gian mà ta cần quan tâm, hay còn gọi là miền quan sát. Ở đây $\mathcal{R} \subset \mathbb{R}^{n_{x}}$, với $\mathbb{R}^{n_{x}}$ là không gian trạng thái của mục tiêu, $n_{x}$ là số chiều của véc tơ trạng thái của mục tiêu.

Ký hiệu $[0, T], T \in \mathbb{R}^{+}$, là khoảng thời gian mà ta cần quan tâm, được gọi là khoảng thời gian của quá trình quan sát. Do các thời điểm quan sát $t_{0}, t_{1}, t_{2}, \ldots, t_{n}$, với $0=$ $t_{0}<t_{1}<\ldots<t_{n}=T$, là rời rạc, nên không mất tính tổng quát, khi nói đến thời điểm thứ $i\left(t_{i}\right)$, chúng ta có thể quy ước $T \in \mathbb{Z}^{+}, t_{i} \in \mathbb{Z}^{+}$và đồng nhất $t_{i}=i, i=0,1, \ldots, T$, trong đó, $t_{0}=0$ là lần quan sát đầu tiên của quá trình quan sát và $t_{n}=T=n$ là lần quan sát cuối cùng.

Số mục tiêu có trong miền $\mathcal{R}$ tại thời điểm $t, t \in[0, T]$, là ngẫu nhiên và ký hiệu là $M_{t}=M_{t}(\omega)$. Giả thiết rằng mục tiêu loại thứ $k$ (để ngắn gọn hơn ta gọi là mục tiêu thứ 
$k), k \in \mathbb{Z}^{+}$, xuất hiện ở vị trí ngẫu nhiên có phân phối đều trong $\mathcal{R}$ tại thời điểm $t_{i}^{k}, t_{i}^{k} \in[0, T]$, và di chuyển (chuyển động) một cách độc lập đối với các mục tiêu khác trong $\mathcal{R}$ đến thời điểm $t_{f}^{k}, t_{f}^{k} \in[0, T]$, thì biến mất. Cũng giả thiết rằng mục tiêu thứ $k$ xuất hiện (tồn tại) với xác suất $p_{k}, 0<p_{k}<1$, và biến mất (không tồn tại) với xác suất $1-p_{k}$. Số mục tiêu tại thời điểm $t, t \in[0, T]$, có trong $\mathcal{R}$, $M_{t}=M_{t}(\omega)$, là biến ngẫu nhiên có phân phối Poisson với tham số $\lambda, \lambda>0$. Các mục tiêu xuất hiện, tồn tại và biến mất một cách độc lập với nhau.

Trong thời gian quan sát, trong miền quan sát có thể có các mục tiêu giả do các clutter hoặc do các thiết bị kỹ thuật và phương pháp quan trắc (đo đạc) gây ra. Cũng tương tự như giả thiết đặt ra với các mục tiêu, giả thiết rằng có $G_{t}=G_{t}(\omega)$ mục tiêu giả trong miền quan sát $\mathcal{R}$ tại thời điểm $t, t \in[0, T]$. Mục tiêu giả thứ $j$ xuất hiện với xác suất $q_{j}, 0<q_{j}<1$, và biến mất với xác suất $1-q_{j}$. Số mục tiêu giả tại thời điểm $t$ trong miền quan sát $\mathcal{R}, G_{t}=G_{t}(\omega)$, là biến ngẫu nhiên Poisson với tham số $\beta, \beta>0$. Các mục tiêu giả xuất hiện, tồn tại và biến mất một cách độc lập với nhau và độc lập với các mục tiêu. Cũng như các mục tiêu, các mục tiêu giả xuất hiện ở vị trí ngẫu nhiên có phân phối đều trong $\mathcal{R}$.

Trong thực tế, các mục tiêu giả có ảnh hưởng như nhau nên ta không cần phân loại các mục tiêu giả. Không mất tính tổng quát, ta coi các mục tiêu giả do clutter gây ra hay do kỹ thuật quan trắc gây ra là một loại với tên gọi là báo động giả (false alarm). Chúng ta coi báo động giả như là một loại mục tiêu đặc biệt.

Nhận xét 1. Tham số $\beta$ hoàn toàn có thể biểu diễn qua các $q_{j}, 1 \leqslant j \leqslant G_{t}$ và tham số $\lambda$ hoàn toàn có thể biểu diễn qua các $p_{k}, 1 \leqslant k \leqslant M_{t}$.

Ký hiệu $X_{t}^{k}, t \in[0, T], k=1,2, \ldots$, là trạng thái của mục tiêu thứ $k$ tại thời điểm $t, X_{t}^{k} \in \mathbb{R}^{n_{x}}, n_{x}$ là số chiều của véc tơ trạng thái. Mô hình chuyển động (mô hình chuyển trạng thái) của mục tiêu thứ $k$ được mô tả bởi hệ động lực tổng quát trong không gian trạng thái $\mathbb{R}^{n_{x}}$ như sau:

$$
X_{t+1}^{k}=F_{k}\left(X_{t}^{k}\right)+V_{t}^{k},
$$

với $F_{k}: \mathbb{R}^{n_{x}} \rightarrow \mathbb{R}^{n_{x}}$ là ánh xạ đo được từ $\mathbb{R}^{n_{x}}$ vào $\mathbb{R}^{n_{x}}$, $V_{t}^{k} \in \mathbb{R}^{n_{x}}$ là nhiễu trắng với ma trận hiệp phương sai là $Q^{k}$, các $V_{t}^{k}$ là không tương quan.

Mô hình quan sát được mô tả bởi

$$
Y_{t}=G\left(X_{t}\right)+W_{t},
$$

với $G: \mathbb{R}^{n_{x}} \rightarrow \mathbb{R}^{n_{y}}, n_{y}$ là số chiều của véc tơ quan sát, $G$ là ánh xạ đo được từ $\mathbb{R}^{n_{x}}$ vào $\mathbb{R}^{n_{y}}, W_{t} \in \mathbb{R}^{n_{y}}$ là nhiễu trắng với ma trận hiệp phương sai là $\mathbb{R}$ và $W_{t}$ không tương quan với các $V_{t}^{k}$.

Nói riêng đối với mục tiêu $k$, từ (2), ta có

$$
Y_{t}^{k}=G\left(X_{t}^{k}\right)+W_{t}
$$

Trong mô hình (1)-(2) ở trên, $V_{t}^{k}$ được gọi là nhiễu hệ thống, $W_{t}$ được gọi là sai số (nhiễu) đo đạc (quan sát).

Ký hiệu $Y(t)=\left\{Y_{t}^{j} \mid j=1,2, \ldots, n_{t}\right\}$ là tập các giá trị quan sát được tại thời điểm $t, n_{t}$ là số lượng các kết quả quan sát được tại thời điểm $t$.

Ký hiệu $Y(0: t)=\cup_{s=0}^{t} Y(s)$ là tập các giá trị quan sát được cho tới thời điểm $t$.

Cần lưu ý rằng tính hữu hạn và bị chặn của $n_{t}$ hiện tại chưa được khẳng định, mà sẽ được nói tới ở phần dưới đây.

Ký hiệu $d(x, y)$, là khoảng cách Euclid trong $\mathcal{R}^{n}$, nghĩa là với $x=\left(x_{1}, \ldots, x_{n}\right) \in \mathcal{R}^{n}$ và $y=\left(y_{1}, \ldots, y_{n}\right) \in$ $\mathcal{R}^{n}$ thì

$$
d(x, y)=\left(\sum_{i=1}^{n}\left(x_{i}-y_{i}\right)^{2}\right)^{\frac{1}{2}} .
$$

Ký hiệu $O_{(O ; r)}, r>0$, là hình cầu mở tâm $O$ bán kính $r, O_{(O ; r)}=\left\{x \in \mathbb{R}^{n}: d(O, x)<r\right\}$, và $\bar{O}_{(O, r)}$, $r>0$, là hình cầu đóng tương ứng, $\bar{O}_{(O ; r)}=\left\{x \in \mathbb{R}^{n}\right.$ : $d(O, x) \leqslant r\}$.

Trong thực tế, do độ phân giải của các cảm biến bị giới hạn, nên trong bài toán MTT xảy ra tình trạng là, với $r>0$ đủ nhỏ nào đó, nếu hai mục tiêu $x$ và $x^{\prime}$ cùng thuộc $O_{(O ; r)}$ (hoặc $\bar{O}_{(O ; r)}$ ) thì dữ liệu quan sát được về chúng $y$ và $y^{\prime}$ tương ứng là như nhau và trùng với dữ liệu quan sát được của tâm điểm $O$. Hiện tượng này trong bài báo này chúng ta gọi là mục tiêu bị che khuất, nghĩa là mục tiêu $x^{\prime}$ bị che khuất bởi mục tiêu $x$ hoặc ngược lại, mục tiêu $x$ bị che khuất bởi mục tiêu $x^{\prime}$. Dưới đây, chúng ta phát biểu giả thiết về mục tiêu bị che khuất (lưu ý là $r$ phụ thuộc vào công cụ và nguyên lý quan sát trong từng bài toán MTT cụ thể).

Giả thiết 1. Tồn tại $r, r>0$, sao cho đối với bài toán (1)(2) nếu $X_{t}^{k}$ và $X_{t}^{l}$ cùng thuộc hình cầu $O_{\left(O_{i} ; r\right)}, O_{\left(O_{i} ; r\right)} \subset$ $\mathbb{R}^{n_{x}}$, thì dữ liệu quan sát được của chúng là như nhau, nghĩa là, nếu $X_{t}^{k} \in O_{\left(O_{i} ; r\right)}$ và $X_{t}^{l} \in O_{\left(O_{i} ; r\right)}$ thì $Y_{t}^{k} \equiv Y_{t}^{l} \equiv Y_{t}^{O_{i}}$, với $Y_{t}^{O_{i}}$ là dữ liệu quan sát được của mục tiêu $X_{t}$ khi $X_{t} \equiv O_{i}$.

Giả thiết 2. Miền quan sát $\mathcal{R}$ là miền đóng và giới nội trong $\mathbb{R}^{n_{x}}$ (theo Metric $d(\cdot, \cdot)$ ).

Từ đó, chúng ta có bổ đề sau.

Bổ đề 1. Với Giả thiết 1 và Giả thiết 2 , tập $n_{t}, t \in[0, T]$ bị chặn đều, nghĩa là, tồn tại $N_{\max }, N_{\max }<+\infty$, sao cho $n_{t} \leqslant N_{\max }$ với mọi $t \in[0, T]$. 
Để chứng minh, chúng ta sử dụng một kết quả về phủ trong lý thuyết tô-pô, được nêu như sau: Xét không gian tô-pô $(\mathcal{X}, \mathcal{T}), \mathcal{M} \subset \mathcal{X}$, nếu $\mathcal{M}$ là tập compact (com-pắc) theo tô-pô $\mathcal{T}$ thì từ mọi phủ mở bất kỳ của $\mathcal{M}$ luôn trích được phủ con hữu hạn. Sau đây là chứng minh bổ đề 1 .

Chứng minh: Xét $\mathcal{X} \equiv \mathbb{R}^{n_{x}}, \mathcal{T}$ là tô-pô cảm sinh bởi Metric $d(\cdot, \cdot)$ trong $\mathbb{R}^{n_{x}}$, từ Giả thiết 2 suy ra $\mathcal{R}$ là tập compact. Xét $\mathcal{P}=\left\{O_{\left(O_{i} ; r\right)}: O_{i} \in\right.$ $\mathcal{R}, r$ là giá trị trong giả thiết 1$\}$. Rõ ràng

$$
\bigcup_{O_{i} \in \mathcal{R}} O_{\left(O_{i} ; r\right)} \supset \mathcal{R} \text {. }
$$

Như vậy $\mathcal{P}$ là một phủ mở của $\mathcal{R}$. Vì $\mathcal{R}$ là compact, theo định lý đã nêu ta suy ra rằng, tồn tại $\mathcal{P}^{*}, \mathcal{P}^{*}=$ $\left\{O_{\left(O_{i_{s}}, r\right)} \mid s=1,2, \ldots, H\right\} \subset \mathcal{P}, H$ hữu hạn, sao cho

$$
\mathcal{R} \subset \bigcup_{s=1}^{H} O_{\left(O_{i_{s}}, r\right)} .
$$

Chúng ta nhận thấy, do có giả thiết 1 nên tại thời điểm $t, t \in[O, T]$ những mục tiêu nằm trong

$$
O_{\left(O_{i_{j}}, r\right)}
$$

thì chỉ có tối đa một giá trị quan sát $Y_{t}^{O_{i_{j}}}$, nằm trong

$$
O_{\left(O_{i_{j}}, r\right)} \cap O_{\left(O_{i_{l}}, r\right)}
$$

thì chỉ có tối đa hai giá trị quan sát $Y_{t}^{O_{i}}, Y_{t}^{O_{i_{l}}}$, nằm trong

$$
O_{\left(O_{i_{j}}, r\right)} \cap O_{\left(O_{i_{l}}, r\right)} \cap O_{\left(O_{i_{s}}, r\right)}
$$

thì chỉ có tối đa ba giá trị quan sát $Y_{t}^{O_{i_{j}}}, Y_{t}^{O_{i_{l}}}, Y_{t}^{O_{i_{s}}}$, v.v.

Như vậy tại thời điểm $t, t \in[0, T]$, số lượng các giá trị quan sát $n_{t}$ của các mục tiêu có thể có trong $\mathcal{R}$ thỏa mãn

$$
n_{t} \leqslant \sum_{s=1}^{H} s \cdot C_{H}^{s}=: N_{\max }
$$

trong đó $C_{H}^{s}$ là tổ hợp chập $s$ của $H$.

Nhận xét 2. Việc khẳng định số các giá trị có thể quan sát được là hữu hạn tại mỗi thời điểm và bị chặn đều với mọi $t, t \in[0, T]$, nhưng ta chưa nói được gì về số lượng của mục tiêu. Vấn đề là trong trường hợp mục tiêu bị che khuất, thì mỗi số liệu quan sát được là của bao nhiêu mục tiêu?

Chúng ta có kết quả dưới đây.

Bổ đề 2. Với mọi $\varepsilon>0$ tùy ý bé, luôn luôn tồn tại $M=$ $M_{\varepsilon}, M_{\varepsilon}<+\infty$, sao cho số mục tiêu có cùng một số đo quan sát $Y_{t}^{O_{k}}$ là $m_{k}$ thỏa mãn

$$
P\left[m_{k}>M_{\varepsilon}\right] \leqslant \varepsilon .
$$

Chứng minh: Xét $O_{\left(O_{k}, r\right)} \in \mathcal{P}^{*}$. Do các mục tiêu xuất hiện tại các vị trí ngẫu nhiên có phân phối đều trong $\mathcal{R}$ và số các mục tiêu có trong $\mathcal{R}, M_{t}=M_{t}(\omega)$, là biến ngẫu nhiên có phân phối Poisson với tham số $\lambda$, ta suy ra số mục tiêu có trong $O_{\left(O_{k}, r\right)} \cap \mathcal{R}$ cũng là biến ngẫu nhiên có phân phối Poisson với tham số

$$
\begin{aligned}
\lambda_{k} & =\frac{V\left(O_{\left(O_{k}, r\right)} \cap \mathcal{R}\right)}{V(\mathcal{R})} \cdot \lambda \\
& \leqslant \frac{V\left(O_{\left(O_{k}, r\right)}\right)}{V(\mathcal{R})} \cdot \lambda,
\end{aligned}
$$

trong đó $V(A), A \subset \mathbb{R}^{n_{x}}$, là số đo "thể tích" của $A$ trong $\mathbb{R}^{n_{x}}$. Như vậy số mục tiêu $m_{k}$ có số đo quan sát là $Y_{t}^{O_{k}}$ có phân phối Poisson với tham số $\lambda_{k}$.

Dễ dàng thấy rằng

$$
\lim _{m \rightarrow+\infty} P\left[m_{k}=m\right]=\lim _{m \rightarrow+\infty} e^{-\lambda_{k}} \cdot \frac{\lambda_{k}^{m}}{m !}=0 .
$$

Từ đó, khẳng định của bổ đề đã được chứng minh.

Từ các bổ đề 1 và 2 , chúng ta có thể giả thiết đối với bài toán MTT đang xét có số mục tiêu không vượt quá $M^{*}$ $\left(M^{*}\right.$ được lấy là giá trị bé nhất có thể để tiết kiệm tính toán).

Giả thiết 3. Số mục tiêu cần quan sát trong $\mathcal{R}$ không vượt quá $M^{*}$ hữu hạn.

\section{PHƯƠNG PHÁP LIÊN KẾT DŨ LIỆU ĐỆ QUY TừNG BƯớC}

\section{Phương pháp liên kết dữ liệu đệ quy}

Yêu cầu của bài toán MTT là, từ các kết quả quan sát (đo) được, xác định (ước lượng) được các quỹ đạo của các mục tiêu. Lưu ý rằng tập hợp các giá trị quan sát được tại thời điểm $t$, tập $Y(t)$, chứa các giá trị quan sát hoặc của mục tiêu này, hoặc của mục tiêu khác hoặc của mục tiêu giả (false alarm) chưa phân định được và mỗi giá trị quan sát đó đại diện cho bao nhiêu mục tiêu bị che khuất cũng chưa rõ. Phương pháp liên kết dữ liệu đệ quy trình bày dưới đây được đưa ra trong hoàn cảnh đó và cho phép khắc phục được các khó khăn nêu trên. Sau đây, chúng ta đưa ra một số định nghĩa.

Định nghĩa 1. Một quỹ đạo của mục tiêu thứ $k$ xuất hiện (bắt đầu) tại thời điểm $t_{i}^{k}, t_{i}^{k} \in[0, T]$ và biến mất (kết thúc) tại thời điểm $t_{f}^{k}, t_{f}^{k} \in[0, T]$ là

$$
X_{\left[t_{i}^{k}, t_{f}^{k}\right]}^{k}=\left\{X_{t}^{k} \mid t_{i}^{k} \leqslant t \leqslant t_{f}^{k} ; t_{i}^{k} \in[0, T] ; t_{f}^{k} \in[0, T]\right\} .
$$

Với $A_{s}$ là các tập hợp, ta sử dụng ký hiệu tích trực tiếp

$$
\bigotimes_{s=1}^{n} A_{s}=\left\{\left(a_{1}, a_{2}, \ldots, a_{n}\right) \mid a_{s} \in A_{s}, s=\overline{1, n}\right\} .
$$


Định nghĩa 2. Một dây chuyền liên kết dữ liệu với thời điểm bắt đầu $t_{i}$ và thời điểm cuối $t_{f}$ và được ký hiệu là $L_{\left[t_{i}, t_{f}\right]}$ là một phần tử của tập tích trực tiếp

$$
\bigotimes_{t=t_{i}}^{t_{f}} Y(t)
$$

nghĩa là

$$
L_{\left[t_{i}, t_{f}\right]}=\left(Y_{t_{i}}^{j_{1}}, Y_{t_{i}+1}^{j_{2}}, \ldots, Y_{t}^{j_{s}}, \ldots, Y_{t_{f}}^{j_{t_{f}-t_{i}+1}}\right)
$$

với $Y_{t}^{j} \in Y(t), t_{i} \leqslant t \leqslant t_{f}$, được gọi là đỉnh tại thời điểm

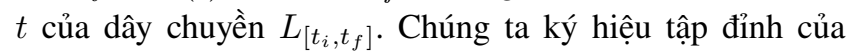
$L_{\left[t_{i}, t_{f}\right]}$ là $D L_{\left[t_{i}, t_{f}\right]}$, nghĩa là

$$
D L_{\left[t_{i}, t_{f}\right]}=\left\{Y_{t_{i}}^{j_{1}}, Y_{t_{i}+1}^{j_{2}}, \ldots, Y_{t_{f}}^{j_{t_{f}-t_{i}+1}}\right\} .
$$

Định nghĩa 3. Dây chuyền $L_{\left[t_{i}, t_{f}\right]}$ được gọi là ảnh của quỹ đạo $X_{\left[t_{i}^{k}, t_{f}^{k}\right]}^{k}$ của mục tiêu thứ $k$ nếu $t_{i}=t_{i}^{k}, t_{f}=t_{f}^{k}$ và giá trị đỉnh $Y_{t}^{j}$ là giá trị quan sát của $X_{t}^{k}$ tại thời điểm $t$ qua mô hình quan sát (2), hoặc cụ thể hơn là (2'), với mọi $t=t_{i}, t_{i+1}, \ldots, t_{f}$.

\section{Nhân xét 3.}

(i) Nếu xác định được dây chuyền ảnh $L_{\left[t_{i}, t_{f}\right]}$ thì việc ước lượng (xác định) quỹ đạo $X_{\left[t_{i}^{k}, t_{f}^{k}\right]}^{k}$ là việc làm đã có nhiều công trình giải quyết đã được công bố, chẳng hạn đơn giản nhất là người ta có thể dùng lọc Kalman để ước lượng quỹ đạo đó $[10,11]$.

(ii) Nếu tại thời điểm $t, t \in[0, T]$, có $m$ dây chuyền cùng nhận $Y_{t}^{j}$ là đỉnh, thì giá trị $Y_{t}^{j}$ là số đo của $m$ mục tiêu (đây là trường hợp có $m$ mục tiêu che khuất lẫn nhau, $\left.m \in \mathbb{N}^{+}\right)$.

(iii) Nếu giá trị $Y_{t}^{s}$ chỉ là đỉnh duy nhất của mọi dây chuyền đi qua nó, thì giá trị $Y_{t}^{s}$ chính là báo động giả tại thời điểm $t$.

Để thuận tiện cho trình bày, chúng ta dùng ký hiệu quy ước sau. Giả sử $a$ là một phần tử nào đó, ta ký hiệu

$$
\{a\}^{\otimes k}=\{\underbrace{a, a, \ldots, a}_{k \text { lần }}\}, \quad k \geqslant 2,
$$

và quy ước trường hợp đặc biệt $\{a\}^{\otimes 0}=\{a\}^{\otimes 1}=\{a\}$. Giả sử $A$ là một tập hợp, $\operatorname{Card}(A)$ là lực lượng (số phần tử) của tập $A$. Với tập $Y(t), t>0$, chúng ta xây dựng hai tập hợp

$$
\begin{gathered}
M[Y(t)]=\bigcup_{j=1}^{n_{t}}\left\{Y_{t}^{j}\right\} \otimes \operatorname{Card}\left(f_{t}^{-1}\left(Y_{t}^{j}\right)\right), \\
\overline{Y_{(t)}}=Y(t) \cup\{\emptyset\} .
\end{gathered}
$$

Định nghĩa 4. Một liên kết dữ liệu từ tập dữ liệu quan sát được tại thời điểm $t-1, t=t_{1}, t_{2}, \ldots, t_{n}$, sang tập dữ liệu quan sát được tại thời điểm $t$ là một ánh xạ $f_{t}$ : $M[Y(t-1)] \rightarrow \overline{Y(t)}$.

Định nghĩa 5. Một lời giải hay còn gọi là một chiến lược liên kết dữ liệu đối với bài toán quan sát đa mục tiêu là họ các ánh xạ $\left\{f_{t} \mid t=t_{1}, t_{2}, \ldots, t_{n}\right\}$.

Từ các định nghĩa trên dễ thấy rằng một lời giải cho ta một họ các dây chuyền ảnh trong tập dữ liệu quan sát $Y(0: T)$.

\section{Nhân xét 4.}

(i) Với $t=t_{0}$, ta dễ thấy $M\left[Y\left(t_{0}\right)\right] \equiv Y\left(t_{0}\right)$. Trong các định nghĩa này chúng ta chỉ đề cập đến trường hợp tại thời điểm ban đầu chúng ta không có thông tin gì về mục tiêu bị che khuất. Bài toán tổng quát chúng ta có thể xét phân phối tiên nghiệm về mục tiêu bị che khuất tại thời điểm $t_{0}$ là $\pi_{0}=\left(\pi_{1}, \pi_{2}, \ldots, \pi_{n_{t_{0}}}\right)$ với các $\pi_{k}, k=1,2, \ldots, n_{t_{0}}$, là các phân phối Poisson với tham số $\lambda_{k}$ tương ứng với $Y_{t_{0}}^{k} \in O_{\left(O_{k}, r\right)} \in \mathcal{P}^{*}$ như đã nêu trong phần chứng minh của bổ đề 2 .

(ii) Giá trị $Y_{t}^{i}$ là đỉnh cuối của dây chuyền nếu $f_{t+1}\left(Y_{t}^{i}\right)=\emptyset$.

(iii) Giá trị $Y_{t}^{j}$ là đỉnh đầu (đỉnh khởi tạo) nếu $\operatorname{Card}\left(f_{t}^{-1}\left(Y_{t}^{j}\right)\right)=0$.

(iv) Giá trị $Y_{t}$ là báo động giả nếu nó vừa là điểm đầu vừa là điểm cuối của dây chuyền.

\section{Lời giải tối ưu từng bước và sự tồn tại của nó}

Dựa theo ý tưởng của suy luận Bayes, chúng ta đưa ra khái niệm lời giải tối ưu theo nghĩa làm cực đại xác suất hậu nghiệm tại mỗi bước cập nhật trạng thái như sau.

Định nghĩa 6. Lời giải $\left\{f_{t}^{*} \mid t=t_{1}, t_{2}, \ldots, t_{n}\right\}$ được gọi là lời giải tối ưu từng bước hay tối ưu cục bộ nếu

$$
P\left[f_{t}^{*} \mid Y(0: t)\right]=\max _{\forall f_{t}} P\left[f_{t} \mid Y(0: t)\right], \quad \forall t .
$$

Ở đây $P\left[f_{t} \mid Y(0: t)\right]$ là xác suất hậu nghiệm của phép gán (ánh xạ) $f_{t}$.

Định lý 1. Với các giả thiết 1 và 2 , bài toán quan sát đa mục tiêu đang xét luôn tồn tại lời giải tối ưu từng bước.

Chúng minh: Từ bổ đề 1 , bổ đề 2 và giả thiết 2 suy ra tại mỗi thời điểm $t, t=t_{1}, \ldots, t_{n}$, ta có $\operatorname{Card}\left(M\left[Y_{t-1}\right]\right)<$ $+\infty$ và $\operatorname{Card}\left(\overline{Y_{t}}\right)<+\infty$. Từ đó suy ra số các ánh xạ có thể có $f_{t}: M\left[Y_{t-1}\right] \rightarrow \overline{Y_{t}}$ là hữu hạn. Do đó, $\left\{P\left[f_{t} \mid Y(0: t)\right]\right\}$ là tập hữu hạn. Từ đó suy ra tồn tại $f_{t}^{*}$ sao cho

$$
P\left[f_{t}^{*} \mid Y(0: t)\right]=\max _{\forall f_{t}} P\left[f_{t} \mid Y(0: t)\right] .
$$


Nhận xét 5. Từ định nghĩa 5 và từ chứng minh của định lý 1 , chúng ta đã thấy rằng lời giải tối ưu từng bước có thể không duy nhất.

Lời giải tối ưu từng bước luôn tồn tại (định lý 1), song việc tìm lời giải đó không đơn giản. Chúng ta có thể xác định được biểu thức giải tích hiển của xác suất hậu nghiệm $P\left[f_{t} \mid Y(0: t)\right]$ (xem [13]) và giải bài toán tìm cực trị của hàm nhiều biến để xác định $f_{t}^{*}$. Nhưng việc đó rất phức tạp và khó khăn trong việc cài đặt thuật toán giải trên máy tính.

Dựa trên ý tưởng của lọc Kalman khi xử lý tín hiệu để ước lượng quỹ đạo của mục tiêu [14-16], ở đây chúng ta đưa ra một quan điểm mới khác để xem xét sự tốt hay không của lời giải theo định nghĩa 5 , cụ thể như sau. Như đã nêu ở định nghĩa 5 , một lời giải xác định một họ các dây chuyền liên kết dữ liệu trong $Y(0: T)$, trong đó mỗi dây chuyền là dây chuyền dữ liệu ảnh của một quỹ đạo xác định của một mục tiêu xác định nào đó. Theo quan điểm của lọc Kalman, chúng ta thấy rằng nếu dùng các giá trị quan sát (dây chuyền dữ liệu ảnh) để ước lượng quỹ đạo thực của mục tiêu tương ứng theo lọc Kalman thì dây chuyền ảnh là tốt nếu như phương sai ước lượng $P(t \mid t)$ là bé nhất và không vượt quá một ngưỡng sai lệch cho trước nào đó. Một lời giải $\left\{f_{t} \mid t=t_{1}, \ldots . t_{n}\right\}$ là tốt nếu như mọi dây chuyền của nó là tốt. Chính xác hơn, chúng ta có định nghĩa sau đây.

Định nghĩa 7. Lời giải $\left\{f_{t}^{* \varepsilon} \mid t=t_{1}, \ldots . t_{n}\right\}$ được gọi là lời giải tối ưu $\varepsilon$-ngưỡng (và gọi tắt là $\varepsilon$-tối ưu) nếu như mọi dây chuyền liên liên kết dữ liệu của nó thỏa mãn các điều kiện sau đây:

(i) Khi sử dụng dữ liệu của dây chuyền để ước lượng quỹ đạo thực của mục tiêu tương ứng theo lọc Kalman thì phương sai ước lượng $P(t \mid t)$ là cực tiểu với mọi $t$ thuộc miền thời gian tồn tại của dây chuyền;

(ii) Giá trị phương sai ước lượng $P(t \mid t)$ nêu trong (i) không vượt quá $\varepsilon, \varepsilon>0$, với mọi $t$ thuộc miền thời gian tồn tại của dây chuyền. Ở đây, $\varepsilon>0, \varepsilon$ cho trước tùy ý bé, được gọi là ngưỡng chấp nhận của lời giải.

Chúng ta đưa ra thuật toán tìm lời giải $\varepsilon$-tối ưu (mà thực chất là tìm họ ánh xạ $\left\{f_{t}^{* \varepsilon} \mid t=t_{1}, \ldots . t_{n}\right\}$ ) trong mục IV tiếp theo.

\section{THUẬT TOÁN TìM LờI GIẢI $\varepsilon$-TỐI ƯU}

\section{Lọc Kalman}

Chúng ta nêu một số nét chính về mô hình và ký hiệu cần sử dụng cho mục đích trình bày kết quả nghiên cứu của bài báo ở mục này (xem Lọc Kalman trong [14-16]). Xét lọc Kalman với thời gian rời rạc đối với mô hình bao gồm: phương trình trạng thái của (1), mô hình quan sát của (2'). Để tránh nhầm lẫn, chúng ta dùng ký hiệu $Z^{t}=$ $\left\{Y_{t_{1}}^{k}, Y_{t_{1}}^{k}, \ldots, Y_{t}^{k}\right\}$ là dãy số liệu quan sát được của mục tiêu thứ $k$ cho tới thời điểm $t$. Trong đó, cần lưu ý rằng $t_{i}^{k} \leqslant t_{1}<t_{2}<\cdots<t \leqslant t_{f}^{k}, Y_{t_{i}}^{k} \in Y\left(t_{i}\right), Z^{t} \subset Y(0: t)$.

Lọc Kalman cho chúng ta ước lượng theo tiêu chuẩn sai số trung bình bình phương bé nhất như sau:

$$
\widehat{X}^{k}(i \mid t)=\arg \min _{X_{i}^{k} \in \mathbb{R}^{n_{x}}} E\left\{\left(X_{i}^{k}-\widehat{X}^{k}\right)\left(X_{i}^{k}-\widehat{X}^{k}\right)^{T} \mid Z^{t}\right\} .
$$

Hiệp phương sai của ước lượng là

$$
P(i \mid t)=E\left\{\left(X_{i}^{k}-\widehat{X}^{k}\right)\left(X_{i}^{k}-\widehat{X}^{k}\right)^{T} \mid Z^{t}\right\} .
$$

Lọc Kalman được thực hiện theo hai bước: dự báo và hiệu chỉnh. Kết quả sau khi áp dụng lọc Kalman (sau bước hiệu chỉnh) cho chúng ta kết quả: $\hat{X}^{k}(t \mid t)$ là ước lượng trạng thái $X_{t}^{k}$ và $P(t \mid t)$ là hiệp phương sai của ước lượng đó (Phương sai của ước lượng).

\section{Thuật toán tìm lời giải $\varepsilon$-tối ưu}

Giả sử cho $\varepsilon$ là một số cho trước tùy ý bé. Giá trị $\varepsilon$ sẽ được gọi là ngưỡng sai lệch. Theo các định nghĩa 4, 5, 6 và 7 , chúng ta xây dựng lời giải $\varepsilon$-tối ưu. Điều đó có nghĩa là chúng ta đi xây dựng họ $\left\{f_{t}^{* \varepsilon} \mid t=t_{1}, t_{2}, \ldots, t_{n}\right\}$ thỏa mãn yêu cầu đòi hỏi. Tư tưởng của thuật toán là kết hợp phương pháp MHT với lọc EKF.

Chúng ta cần một số khái niệm và ký hiệu sau đây. Xét tại thời điểm $t, t=t_{0}, t_{1}, \ldots, t_{n}$ nào đó. Ký hiệu $L_{l}\left[t^{-}, Y_{t}^{i}\right]$, $0 \leqslant l \leqslant \operatorname{Card}\left(f_{t}^{-1}\left(Y_{t}^{i}\right)\right), Y_{t}^{i} \in Y(t)$ là dây chuyền thứ $l$ có đỉnh cuối tại thời điểm $t$ là $Y_{t}^{i}$.

Trong trường hợp $\operatorname{Card}\left(f_{t}^{-1}\left(Y_{t}^{i}\right)\right)=0$, tương đương với $l=0$, nghĩa là $Y_{t}^{i}$ là số đo mới xuất hiện chưa được gắn với dây chuyền nào trước đó. Nó có thể là điểm khởi đầu (đỉnh đầu) cho một dây chuyền mới là ảnh của quỹ đạo của mục tiêu mới xuất hiện nào đó. Nó cũng có thể là điểm cô lập (hay số đo của FA) mà sẽ được kết luận khi thực hiện thuật toán sau mốc thời gian $t+1$.

Ký hiệu $D L_{l}\left[t^{-}, Y_{t}^{i}\right]$ là tập các đỉnh của dây chuyền $L_{l}\left[t^{-}, Y_{t}^{i}\right]$ (kể cả đỉnh cuối tính đến thời điểm $t$ là $Y_{t}^{i}$ ), $0 \leqslant l \leqslant \operatorname{Card}\left(f_{t}^{-1}\left(Y_{t}^{i}\right)\right)$.

Với $1 \leqslant j \leqslant n_{t+1}$, ký hiệu

$$
\begin{aligned}
Z^{t+1}(j) & =D L\left[t^{-}, Y_{t}^{i}\right] \cup\left\{Y_{t+1}^{j}\right\} \\
& =\left\{Y_{s}^{h} \in L\left[t^{-}, Y_{t}^{i}\right] \mid 1 \leqslant h \leqslant s, s \leqslant t\right\} \cup\left\{Y_{t+1}^{j}\right\} .
\end{aligned}
$$

Trong bài toán MTT, các hàm $F_{k}(\cdot)$ trong mô hình biến đổi trạng thái là chưa biết. Trong thực tế người ta có thể có một số thông tin tiên nghiệm nào đó hay có thể có một số dự báo nào đó về dạng, loại hoặc tính chất của các hàm này. Những thông tin tiên nghiệm và dự báo về các hệ động lực trong mô hình biến đổi trạng thái (quá trình chuyển động) của mục tiêu $X_{t}^{k}$ được biểu diễn bởi họ $\left\{F_{\theta}^{k} \mid \theta \in \Theta\right\}$. 
Thực tế không cần phân định một quỹ đạo cụ thể này là quỹ đạo của mục tiêu thứ mấy, nên không mất tính tổng quát người ta coi $F_{\theta}^{k}$ không phụ thuộc vào $k$, nghĩa là, $F_{\theta}^{k}=F_{\theta}, \theta \in \Theta$.

Ký hiệu $\mathcal{F}=\left\{F_{\theta} \mid \theta \in \Theta\right\}$ và gọi là tập thông tin tiên nghiệm và dự báo mô tả về hệ động lực có thể có của các mục tiêu cần quan sát. Cần lưu ý rằng nếu không có thông tin tiên nghiệm hay dự báo nào thì khi xét phải xét với mọi hàm là ánh xạ đo được: $F_{\theta}: \mathbb{R}^{n_{x}} \rightarrow \mathbb{R}^{n_{x}}$. Chúng ta nghiên cứu chỉ với giả thiết $\operatorname{Card}(\Theta)<+\infty$. Thông tin tiên nghiệm và dự báo càng tốt thì $\operatorname{Card}(\Theta)$ càng nhỏ và số lượng tính toán trong thuật toán càng giảm đi.

Khi sử dụng lọc Kalman trong tính toán liên quan chặt chẽ với mô hình biến đổi trạng thái $F$, mô hình quan sát $G$ và tập dữ liệu quan sát $Z^{t}$. Song do mô hình quan sát $G$ là không thay đổi và đã biết nên chúng ta không cần chỉ rõ sự phụ thuộc vào $G$. Khi thực hiện bài toán lọc, theo (1) và (2'), với $F=F_{\theta}$, bộ dữ liệu quan sát $Z^{t+1}(j)$, ta tính được phương sai hiệu chỉnh ở bước $t+1$ và ký hiệu là $P_{l i j}^{F_{\theta}}(t+1 \mid t+1)$.

Để đơn giản trong trình bày cũng như để thuận tiện trong cài đặt, chúng tôi trình bày thuật toán tìm lời giải chấp nhận được $\varepsilon$-tối ưu theo các bước như dưới đây. Sơ đồ khối xử lý thuật toán được mô tả trong hình 1 .

Bước 1: Chọn thời điểm hiện tại $t, 1 \leqslant t \leqslant T$ (thực hiện tuần tự $t=t_{0}, t_{1}, \ldots, t_{n}-1$, nếu $t=t_{k}$ thì $t+1=t_{k+1}$ ).

Tạo tập dữ liệu quan sát ở thời điểm $t+1$ (nếu $t+1 \leqslant T$ )

$$
Y(t+1)=\left\{Y_{t+1}^{j} \mid 1 \leqslant j \leqslant n_{t+1}\right\} .
$$

Nếu không có số liệu thực tế từ bài toán MTT cụ thể thì tập $Y(t+1)$ được tạo bằng mô phỏng theo phân phối Poisson.

Bước 2: Chọn $i, 1 \leqslant i \leqslant n_{t}$, tương đương với xác định $Y_{t}^{i}$.

Với mỗi $l, 0 \leqslant l \leqslant \operatorname{Card}\left(f_{t}^{-1}\left(Y_{t}^{i}\right)\right)$ được xét tuần tự với

$$
l=0,1, \ldots, \text { Card }\left(f_{t}^{-1}\left(Y_{t}^{i}\right)\right),
$$

sử dụng lọc Kalman tính $P_{l i j}^{F_{\theta}}(t+1 \mid t+1)$ ứng với mọi $j$, $1 \leqslant j \leqslant n_{t+1}$, nghĩa là ứng với mọi

$$
Z^{t+1}(j)=D L_{l}\left[t^{-}, Y_{t}^{i}\right] \cup\left\{Y_{t+1}^{j}\right\}, \quad 1 \leqslant j \leqslant n_{t+1} .
$$

Tính

$$
\delta_{l i}=\min _{1 \leqslant j \leqslant n_{t+1}}\left\{\min _{\theta \in \Theta} P_{l i j}^{F_{\theta}}(t+1 \mid t+1)\right\} .
$$

So sánh $\delta_{l i}$ với ngưỡng $\varepsilon$ cho trước. Nếu $\delta_{l i} \geqslant \varepsilon$, ta kết luận dây chuyền $L_{l}\left[t^{-}, Y_{t}^{i}\right]$ kết thúc tại đỉnh cuối $Y_{t}^{i}$, tương đương với $f_{t+1}^{* \varepsilon}\left(Y_{t}^{i}\right)=\varnothing$. Nếu $\delta_{l i}<\varepsilon$, ký hiệu

$$
\left(j_{*}, \theta_{*}\right)=\arg \min _{1 \leqslant j \leqslant n_{t+1}}\left\{\min _{\theta \in \Theta} P_{l i j}^{F_{\theta}}(t+1 \mid t+1)\right\} .
$$

Khi đó dây chuyền $L_{l}\left[t^{-}, Y_{t}^{i}\right]$ được nối tiếp từ đỉnh $Y_{t}^{i}$ sang đỉnh $Y_{t+1}^{j_{*}}$ theo quỹ đạo chuyển trạng thái $F_{\theta_{*}}$ và để rõ hơn ta ký hiệu thêm chỉ số $F_{\theta_{*}}^{(t+1)}$. Nghĩa là với $Y_{t}^{i}$ là đỉnh của dây chuyền $L_{l}\left[t^{-}, Y_{t}^{i}\right]$ ta có

$$
f_{t+1}^{* \varepsilon}\left(Y_{t}^{i}\right)=Y_{t+1}^{j_{*}} \text {. }
$$

Cần lưu ý rằng $f_{t+1}: M[Y(t)] \rightarrow \bar{Y}_{t+1}$.

Bước 3: Kiểm tra $l$. Nếu $l<\operatorname{Card}\left(f_{t}^{-1}\left(Y_{t}^{i}\right)\right)$ thì quay lại làm tiếp với $l:=l+1$. Còn nếu $l=\operatorname{Card}\left(f_{t}^{-1}\left(Y_{t}^{i}\right)\right)$ thì kiểm tra $i$. Nếu $i<n_{t}$ thì quay lại bước 2 với việc thay $i:=i+1$, còn nếu $i=n_{t}$ thì chuyển sang bước 4 .

Bước 4: Kiểm tra $t+1$. Nếu $t+1<T=t_{n}$, quay lại bước 1 với việc thay $t:=t+1$, còn nếu $t+1 \geqslant T$ thì kết thúc thuật toán.

Chú ý: Trong bước 2 , chúng ta cần nhấn mạnh và nói rõ hơn vấn đề sau.

Khi xét tới dây chuyền $L_{l}\left[t^{-}, Y_{t}^{i}\right]$, ta giả sử dây chuyền này xuất phát từ đỉnh đầu $Y_{s}^{m}$ tại thời điểm $s, s \in[0, T]$ nào đó, khi đó ta hoàn toàn xác định

$$
[s, t]=\left[t_{s}, t_{s+1}\right] \cup\left(t_{s+1}, t_{s+2}\right] \cup \ldots \cup\left(t_{k}, t\right] .
$$

Do thuật toán là đệ quy tuần tự, nên khi xét đến thời điểm $t$ thì tất cả các quỹ đạo chuyển trạng thái tối ưu trên từng khoảng giữa các bước là $F_{t^{*}}^{h}, h \leqslant t$, đã được xác định. Ta xây dựng "hàm dán" như sau:

$F_{L S}(\bullet)=\left\{F_{t^{*}}^{h}(\bullet)\right.$, với $\left.\bullet \in(h-1, h] ; t_{q} \leqslant h-1 ; h \leqslant t\right\}$.

Hàm $F_{L S}$ mô tả hệ động lực của mục tiêu có ảnh quỹ đạo là dây chuyền $L_{l}\left[t^{-}, Y_{t}^{i}\right]$. Khi thực hiện bài toán lọc theo (1) và (2') trong bước 2 , hệ động lực $F$ được thực hiện là

$$
F(\bullet)= \begin{cases}F_{L S}(\bullet), & \bullet \leqslant t, \\ F_{\theta}(\bullet), & t \leqslant \bullet \leqslant t+1 .\end{cases}
$$

Vì lẽ $F_{L S}(\bullet)$ đã được xác định nên trong ký hiệu ta chỉ ký hiệu phụ thuộc $F_{\theta}$ (trong ký hiệu $P_{l i j}^{F_{\theta}}(t+1 \mid t+1)$ ).

\section{THẢO LUẬN}

Các phương pháp tiên tiến hiện hành như đã nêu lên trong mục I (GNN, JPDA, MHT, NNJPDA), đều không đề cập đến khái niệm và xét trường hợp mục tiêu bị che khuất. Do đó, trong trường hợp mục tiêu bị che khuất, các thuật toán này không giải quyết được tình trạng có bị mất mục tiêu, có bị mất quỹ đạo bám, liên quan đến hạn chế về những xử lý trong cập nhật phần tử đổi mới (trạng thái mới của mục tiêu).

Bài báo này đã giải quyết được vấn đề đó dựa trên phương pháp liên kết dữ liệu thông qua hệ thống ánh xạ được xây dựng đệ quy từng bước. Hệ thống ánh xạ này không chỉ quan tâm tới bản thân số liệu quan sát mà còn tính đến cả lịch sử quỹ đạo quá khứ có thể có của số liệu (bao gồm tập dữ liệu $D L_{l}\left[t^{-}, Y_{t}^{i}\right]$, thông tin lịch sử dáng điệu chuyển 


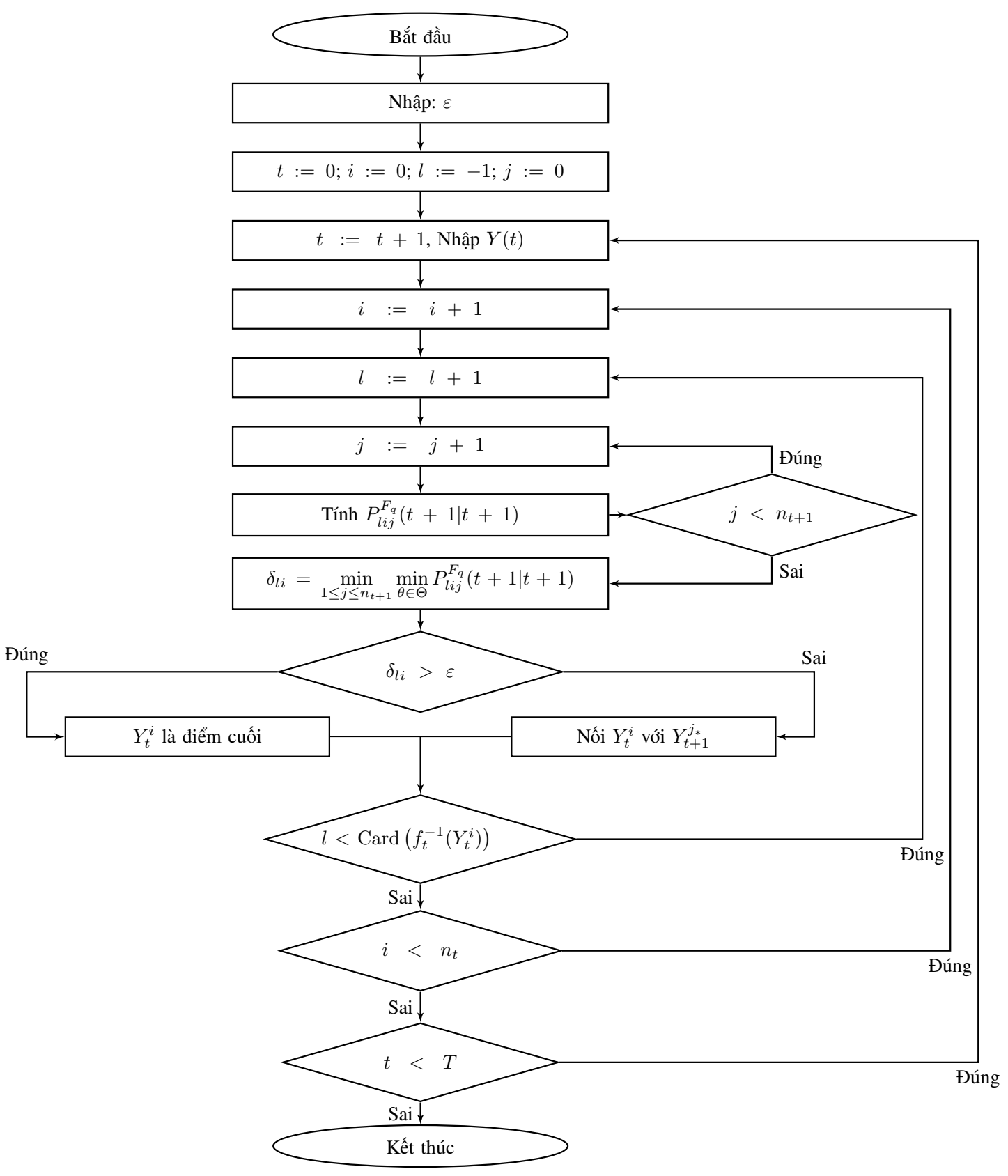

Hình 1. Sơ đồ khối xử lý thuật toán.

trạng thái $F_{L S}(\bullet)$. Với thuật toán tìm lời giải $\epsilon$ - tối ưu, chúng ta sẽ ước lượng được quỹ đạo của mục tiêu thông qua dữ liệu quan sát của dây truyền dữ liệu ảnh (lời giải tối ưu ở đây được hiểu theo nghĩa làm cực đại xác suất hậu nghiệm tại mỗi bước).

Mô hình mà bài báo nghiên cứu có rất nhiều ứng dụng trong nhiều lĩnh vực cả dân sự lẫn quân sự. Một ví dụ minh họa cho việc sử dụng phương pháp đề xuất là trong hệ thống theo dõi phòng không. Mục tiêu quan sát là các máy bay cần theo dõi. Hệ thống quan sát là hệ thống ra đa phòng không trong đó thuật toán đề xuất có thể được cài đặt để theo dõi sự biến mất của mục tiêu khi máy bay bị bắn hạ hoặc ra ngoài vùng tác chiến. Việc bám quỹ đạo của mục tiêu được thể hiện qua tiêu đồ tham mưu của bộ chỉ huy.

Bài báo này tập trung xây dựng phương pháp và chứng minh tính đúng đắn bằng toán học, cũng như đề xuất giải thuật tương ứng. Do giới hạn số trang, việc nghiên cứu thực nghiệm mô phỏng bằng dữ liệu mô phỏng và dữ liệu thực tiễn sẽ được quan tâm trong những công trình tiếp theo. 


\section{KẾT LUẬN}

Bài báo trình bày một số kết quả nghiên cứu mới về bài toán MTT trong điều kiện có thể xảy ra tình trạng mục tiêu bị che khuất, gây nên mất mục tiêu, mất quỹ đạo bám mà các nghiên cứu hiện hành chưa giải quyết được. Trước hết, chúng tôi đã xây dựng phương pháp liên kết dữ liệu đệ quy bằng hệ thống ánh xạ không chỉ tính đến bản thân dữ liệu quan sát mà còn tính đến cả lịch sử quỹ đạo của dữ liệu đó. Hơn nữa, theo quan điểm của suy luận Bayes, đưa ra khái niệm lời giải tối ưu từng bước làm cực đại xác suất hậu nghiệm và chứng minh sự tồn tại của nó đối với phương pháp liên kết dữ liệu như đã đưa ra. Cuối cùng, chúng tôi đã kết hợp với lọc Kalman, đưa ra khái niệm lời giải tối ưu $\varepsilon$-ngưỡng (gọi tắt là $\varepsilon$-tối ưu) và đưa ra thuật toán tìm lời giải đó đối với phương pháp liên kết dữ liệu đã đưa ra.

\section{TÀI LIÊU THAM KHẢO}

[1] Y. Bar-Shalom, P. K. Willett, and X. Tian, Tracking and data fusion. YBS Publishing, CT, 2011.

[2] S. Blackman and R. Popoli, Design and analysis of modern tracking systems. Artech House, Norwood, MA, 1999.

[3] J. Yi, Y. Du, F. Liang, and C. Zhou, "An auto-tracking algorithm for mesoscale eddies using global nearest neighbor filter," Limnology and Oceanography: Methods, vol. 15, no. 3, pp. 276-290, 2017.

[4] Y. Bar-Shalom and X.-R. Li, Multitarget-multisensor tracking: principles and techniques. YBS Publishing, CT, 1995.

[5] K.-C. Chang and Y. Bar-Shalom, "Joint probabilistic data association for multitarget tracking with possibly unresolved measurements and maneuvers," IEEE Transactions on Automatic Control, vol. 29, no. 7, pp. 585-594, 1984.

[6] S. Yang, K. Thormann, and M. Baum, "Linear-time joint probabilistic data association for multiple extended object tracking," in 2018 IEEE 10th Sensor Array and Multichannel Signal Processing Workshop (SAM), 2018, pp. 6-10.

[7] S. S. Blackman, "Multiple hypothesis tracking for multiple target tracking," IEEE Aerospace and Electronic Systems Magazine, vol. 19, no. 1, pp. 5-18, 2004.

[8] M. Mallick, S. Coraluppi, C. Carthel, V. Krishnamurthy, and B. Vo, "Multitarget tracking using multiple hypothesis tracking," in Integrated Tracking, Classification, and Sensor Management: Theory and Applications. Wiley Online Library, 2012, ch. 2, pp. 165-201.

[9] D. Reid, "An algorithm for tracking multiple targets," IEEE transactions on Automatic Control, vol. 24, no. 6, pp. 843 854, 1979.

[10] Z. Zhang, K. Fu, X. Sun, and W. Ren, "Multiple target tracking based on multiple hypotheses tracking and modified ensemble Kalman filter in multi-sensor fusion," Sensors, vol. 19 , no. 14 , p. $3118,2019$.

[11] W. D. Blair and M. Brandt-Pearce, "NNJPDA for tracking closely spaced Rayleigh targets with possibly merged measurements," in SPIE Conference on Signal and Data Processing of Small Targets, vol. 3809, 1999, pp. 396-408.

[12] S. Varghese, P. Sinchu, and D. S. Bhai, "Tracking crossing targets in passive sonars using NNJPDA," Procedia Computer Science, vol. 93, pp. 690-696, 2016.

[13] N. Hang and N. Nam, "Bài toán quan sát đa mục tiêu: Sự tồn tại lời giải tối ưu và thuật toán Kalman tìm nghiệm theo ngường xác định," Tạp chí nghiên cứu Khoa hoc Công nghệ Quân sư, no. 46, pp. 149-157, 2016.
[14] H. Durrant-Whyte et al., "Introduction to estimation and the Kalman filter," Autralia, Tech. Rep., 2001, version 2.2.

[15] S. Särkkä, Bayesian filtering and smoothing. Cambridge University Press, 2013.

[16] S. Yang and M. Baum, "Extended Kalman filter for extended object tracking," in 2017 IEEE International Conference on Acoustics, Speech and Signal Processing (ICASSP), 2017, pp. 4386-4390.

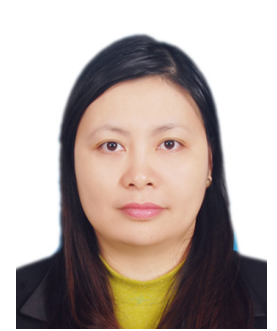

Nguyễn Thị Hằng sinh năm 1975. Bà tốt nghiệp Đại học ngành Toán, tại Trường Đại học Sư phạm Hà Nội năm 1996, Thạc sỹ chuyên ngành Xác suất Thống kê, tại Trường Đại học Khoa học Tự nhiên, Đại học Quốc gia Hà Nội, năm 2000. Hiện công tác tại Bộ môn Toán, Khoa Khoa học Cơ bản, Trường Đại học Mỏ - Địa chất. Lĩnh vực nghiên cứu bao gồm mô hình tuyến tính nhiều biến, mô hình chuỗi thời gian, tiếp cận Bayes và lọc Bayes, bài toán theo dõi đa mục tiêu di động. 